\title{
A new gauge-invariant double copy for heavy-mass effective theory
}

\author{
Andreas Brandhuber, Gang Chen, Gabriele Travaglini and Congkao Wen \\ Centre for Research in String Theory, School of Physics and Astronomy, \\ Queen Mary University of London, \\ Mile End Road, London E1 4NS, United Kingdom \\ E-mail: a.brandhuber@qmul.ac.uk, g.chen@qmul.ac.uk, \\ g.travaglini@qmul.ac.uk, c.wen@qmul.ac.uk
}

ABSTRACT: We propose a new form of the colour-kinematics/double-copy duality for heavymass effective field theories, which we apply to construct compact expressions for tree amplitudes with heavy matter particles in Yang-Mills and in gravity to leading order in the mass. In this set-up, the new BCJ numerators are fixed uniquely and directly written in terms of field strengths, making gauge invariance manifest. Furthermore, they are local and automatically satisfy the Jacobi relations and crossing symmetry. We construct these BCJ numerators explicitly up to six particles. We also discuss relations of the BCJ numerators in the heavy-mass effective theory with those in pure Yang-Mills amplitudes.

KEYwords: Gauge Symmetry, Scattering Amplitudes

ArXiv ePrint: 2104.11206 


\section{Contents}

1 Introduction 1

2 Elements of heavy-mass effective theory 3

3 Traditional double copy construction 5

4 A novel double copy from gauge-invariant BCJ numerators $\quad 7$

4.1 Background for the colour-kinematics algebra from fusion rules $\quad 7$

$\begin{array}{lll}4.2 & \text { General method to construct the pre-numerator } & 9\end{array}$

5 Applications of the new double copy 11

$\begin{array}{lll}5.1 \text { Three-point numerator } & 11\end{array}$

5.2 Four-point numerator 11

$\begin{array}{lll}5.3 & \text { Five-point numerator } & 12\end{array}$

$\begin{array}{lll}5.4 \text { Six-point numerator } & 14\end{array}$

6 Local BCJ numerators for pure Yang-Mills theory 15

$\begin{array}{lll}7 & \text { Conclusions } & 17\end{array}$

\section{Introduction}

Problems in physics where the typical scale of the momenta is much smaller than the masses of the particles at play can be conveniently described using effective field theories. The prototype example is heavy-quark effective theory [1-4], where one is interested in studying the dynamics of heavy quarks exchanging momenta which are typically much smaller than their mass. The effective description is particularly convenient in that it reveals symmetries that are not present in the QCD Lagrangian, leading to a velocity superselection rule. Another example is the dynamics of black holes, which in many situations of practical relevance can be effectively considered as heavy pointlike particles exchanging momenta which are much smaller than their mass. In this context, the computation of the classical part of observable quantities, such as the deflection angle of a massless particle by a heavy black hole, is of particular interest. The classical limit is then reached by scaling the momentum of the exchanged massless gravitons as $\vec{q}=\hbar \vec{k}$ and taking the $\hbar \rightarrow 0$ limit while keeping the wavevector $\vec{k}$ fixed, together with the masses and momenta of the black holes [5]. An approach to the effective field theory describing heavy scalars and fermions minimally coupled to gravity in the spirit of the heavy-quark effective theory was pursued in [6], with applications to Feynman-diagram computations of four-point amplitudes at one loop. 
Recently, considerable effort has been devoted to applying modern amplitude methods to the computation of observable quantities in general relativity. This approach leads to significant conceptual as well as practical simplifications, since all steps of the computation are manifestly gauge invariant. Examples of this include applications of unitarity at one [7-16], two [17-19] and three loops [20] to the computation of bending angles, and classical and quantum corrections to the gravitational potential. An important tool in the amplitude arsenal is the colour-kinematics and double-copy duality [21-23], which makes explicit an intriguing interplay between colour and kinematics of amplitudes in Yang-Mills and other theories [24-38]. This also allows one to construct gravity amplitudes from YangMills ones once the latter are expressed in a so-called BCJ form, in a similar spirit to the KLT relations [39]. Further applications of this duality include [40-48] and [49-59] for the construction of BCJ numerators at tree and loop level, respectively.

Intriguingly, there is strong evidence that underlying the colour-kinematics duality there must be a kinematic algebra obeyed by the BCJ numerators. This algebra was originally discovered in the self-dual sector in [60,61], with recent important efforts to understand it beyond that sector in [62, 63]. We also mention the work of [36, 38, 64-71] where a Lagrangian or geometric understanding of the duality was sought. With a view of applying the colour-kinematics duality to the problem of black hole scattering, one is interested in amplitudes containing two massive particles as well as gravitons, which in general can also be constructed via the double copy [72-79]. The double copy was also directly applied to the heavy-quark effective theory in [80] for particles of spin $s \leq 1$, in particular constructing three- and four-point amplitudes with two heavy spinning particles and one and two gravitons.

In this paper we systematically apply the method of [62] to obtain compact expressions for amplitudes with two massive scalars and an arbitrary number of gluons or gravitons via the double copy in a heavy-mass effective theory (HEFT) at leading order in an inverse mass expansion. These will be used in [81] to compute loop amplitudes of two heavy scalars with graviton interactions, from which one can extract classical quantities such as the bending angle and corrections to the Newtonian potential. Our HEFT amplitudes will enter the relevant unitarity cuts, crucially simplifying the loop integrations because of their special features [81].

In the approach of [62], one introduces vector and tensor currents representing the generators of the kinematic algebra, with a fusion rule among them. This fusion rule was completely determined in [62, 63] in Yang-Mills in the MHV and NMHV sectors for arbitrary multiplicity, with explicit examples up to eight particles. ${ }^{1}$ In the present work we apply these ideas to the HEFT, computing general amplitudes up to six points and in any number of dimensions. Importantly, these are the necessary ingredients to compute the two-to-two scattering amplitude of two heavy scalars up to three loops.

This new approach is very powerful in that it leads to BCJ numerators automatically satisfying the Jacobi relations and crossing symmetry, under the assumption that the fusion

\footnotetext{
${ }^{1}$ In general dimensions, by MHV amplitude in pure Yang-Mills we mean one whose numerator has the schematic form $(\epsilon \cdot \epsilon) \prod(\epsilon \cdot p)$, whereas NMHV corresponds to $(\epsilon \cdot \epsilon)^{2} \prod(\epsilon \cdot p)$, and so on.
} 
rule is associative. In practice, one starts by building a function called the pre-numerator (which, despite its name, can have denominators from propagators of the heavy particles), which is written as a product of many currents multiplied using the fusion rule. The BCJ numerators are then obtained by taking appropriate anti-symmetrisations of the external particles in the pre-numerator, thereby forming nested commutator structures associated to cubic graphs. With the assumption of associativity of the fusion rule, this operation produces BCJ numerators that automatically satisfy colour-kinematics duality. We also stress that only a subset of all possible cubic graphs appears in our construction, namely those where the two massive particles connect via a single cubic vertex to the rest of the graph. Each cubic graph is in one-to-one correspondence with a nested commutator.

An additional important feature of our work is that the BCJ numerators we obtain for each cubic graph are uniquely determined, manifestly gauge invariant (i.e. written in terms of field strengths) and local with respect to the massless gluons or gravitons. This is to be contrasted with the situation in Yang-Mills amplitudes, where the BCJ numerators are in general neither gauge invariant nor unique. As a byproduct of our analysis, we also show how to derive BCJ numerators in pure Yang-Mills by taking appropriate limits of our BCJ numerators.

The rest of the paper is organised as follows. In the next section and in section 3 we briefly review basic properties of heavy-quark effective theory and of the double copy, respectively. In section 4 we present the construction of amplitudes from the novel double copy. We briefly review the approach based on fusion rules, which we then discuss in the context of our HEFT. In particular we discuss the construction of the pre-numerator from fusion rules and from an ansatz, in terms of which the BCJ numerators are expressed. In section 5 we systematically treat cases up to six particles. Importantly, we find unique, gauge-invariant BCJ numerators. In section 6 we briefly discuss how to obtain pure YangMills numerators from HEFT numerators in a particular limit. We present our conclusions and an outlook on future work in section 7 .

\section{Elements of heavy-mass effective theory}

Heavy-quark effective theory [1-4] plays an important role in hadron physics. In this set-up, the momentum of an incoming heavy quark is written as

$$
p^{\mu}=m v^{\mu},
$$

where $m$ is the heavy mass of the quark and $v^{2}=1$, which after an interaction with a soft particle becomes

$$
p^{\mu}=m v^{\mu}+k^{\mu} .
$$

In QCD, the momentum $k^{\mu}$ would be taken to be of order $\Lambda_{\mathrm{QCD}} \ll m$. We are ultimately interested in applications to classical physics (discussed in the companion paper [81]), in which case it is convenient to think of the residual soft momentum as being rescaled as 
$k^{\mu}=\hbar \bar{k}^{\mu}[5]$, keeping $\bar{k}$ fixed as $\hbar \rightarrow 0$. If $p$ is the momentum of an on-shell state, for example an outgoing heavy quark, we also get the constraint $v \cdot k=-k^{2} /(2 m)$, which implies that $v \cdot k=0$ in the large-mass limit. The leading terms of the effective Lagrangian are

$$
\mathcal{L}_{\text {eff }}=-\frac{1}{4}\left(F_{\mu \nu}^{a}\right)^{2}+i \bar{Q}_{v} v \cdot(\partial-i g A) \frac{1+\psi}{2} Q_{v}+\mathcal{O}(1 / m),
$$

where for external fermion states one also has $\psi Q_{v}=Q_{v}$. If one ignores the $\mathcal{O}(1 / m)$ terms, the velocity $v$ and the polarisation of $Q_{v}$ are conserved. The Feynman rules for the fermion propagator and vertex are easily found to be

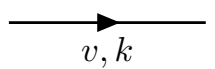

$\mu, p_{2}$

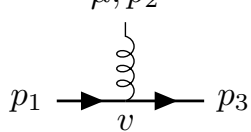

$$
\frac{i}{v \cdot k+i \varepsilon} \frac{1+\psi}{2}
$$

$$
i g T^{a} v_{\mu} \frac{1+\psi}{2}
$$

which are accompanied by the standard Feynman rules for gauge fields. Importantly, the leading-order contribution in the heavy-mass limit is universal, that is the heavy quark field can be replaced by a heavy scalar or vector field without changing the amplitudes.

One can now use these Feynman rules to compute directly HEFT amplitudes, at least for a small number of legs. For higher multiplicities this becomes very involved, and we will introduce more efficient techniques in the next two sections. ${ }^{2}$ The three-point amplitude is given by

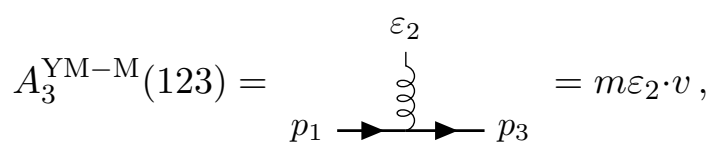

while the four-point amplitude is

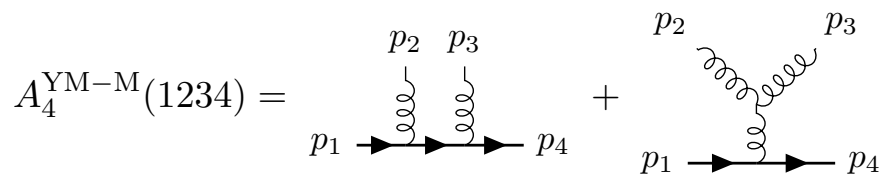

$$
\begin{aligned}
& =2 m\left(-\frac{\varepsilon_{2} \cdot p_{3} v \cdot \varepsilon_{3}}{s_{23}}-\frac{\varepsilon_{2} \cdot \varepsilon_{3} v \cdot p_{2}}{s_{23}}+\frac{\varepsilon_{3} \cdot p_{2} v \cdot \varepsilon_{2}}{s_{23}}+\frac{v \cdot \varepsilon_{2} v \cdot \varepsilon_{3}}{2 v \cdot p_{2}}\right) .
\end{aligned}
$$

The Feynman diagrams contributing to the five-point amplitude are
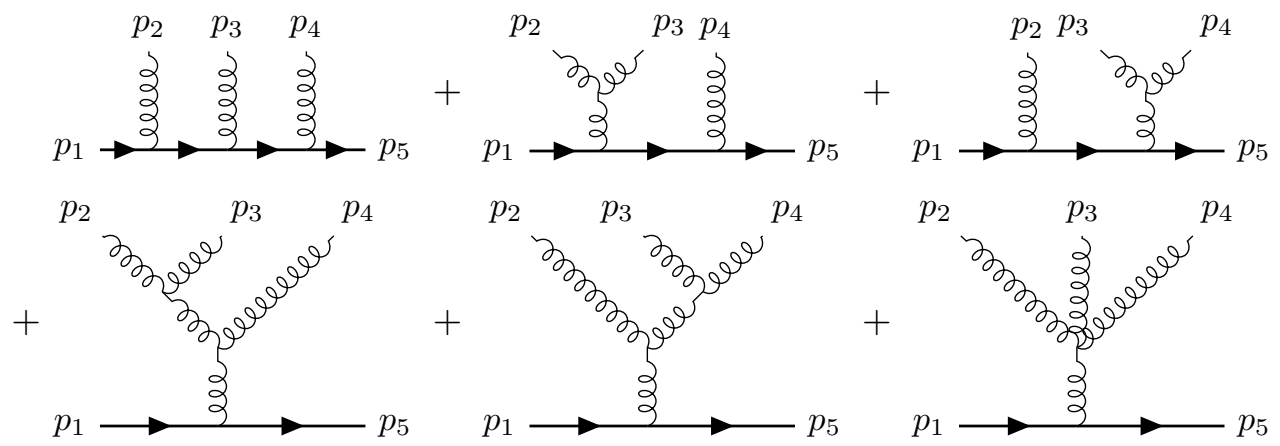

\footnotetext{
${ }^{2}$ In the following we quote colour-ordered amplitudes and drop an ubiquitous factor of $i g^{n-2}$.
} 
leading to the — somewhat lengthy — result

$$
\begin{aligned}
& A_{5}^{\mathrm{YM}-\mathrm{M}}(12345)=4 m\left[\frac{v \cdot \varepsilon_{2} v \cdot \varepsilon_{3} v \cdot \varepsilon_{4}}{4 v \cdot p_{2} v \cdot p_{23}}+\frac{v \cdot \varepsilon_{4}\left(\frac{1}{2} \varepsilon_{2} \cdot \varepsilon_{3} v \cdot\left(p_{3}-p_{2}\right)+\varepsilon_{3} \cdot p_{2} v \cdot \varepsilon_{2}-\varepsilon_{2} \cdot p_{3} v \cdot \varepsilon_{3}\right)}{2 s_{23} v \cdot p_{23}}\right. \\
& +\frac{v \cdot \varepsilon_{2}\left(-\varepsilon_{3} \cdot p_{4} v \cdot \varepsilon_{4}-\frac{1}{2} \varepsilon_{3} \cdot \varepsilon_{4} v \cdot p_{3}+\frac{1}{2} \varepsilon_{3} \cdot \varepsilon_{4} v \cdot p_{4}+\varepsilon_{4} \cdot p_{3} v \cdot \varepsilon_{3}\right)}{2 s_{34} v \cdot p_{2}} \\
& +\frac{1}{2 s_{23} s_{234}}\left(v \cdot \varepsilon_{4}\left(\left(\frac{s_{24}-s_{34}}{2}\right) \varepsilon_{2} \cdot \varepsilon_{3}-2 \varepsilon_{2} \cdot p_{4} \varepsilon_{3} \cdot p_{2}+2 \varepsilon_{2} \cdot p_{3} \varepsilon_{3} \cdot p_{4}\right)\right. \\
& +\frac{s_{23}}{2}\left(\varepsilon_{2} \cdot \varepsilon_{4} v \cdot \varepsilon_{3}-\varepsilon_{3} \cdot \varepsilon_{4} v \cdot \varepsilon_{2}\right)-\varepsilon_{4} \cdot p_{23}\left(\varepsilon_{2} \cdot p_{3} v \cdot \varepsilon_{3}-\varepsilon_{3} \cdot p_{2} v \cdot \varepsilon_{2}\right) \\
& \left.+2\left(v \cdot p_{23} \varepsilon_{3} \cdot \varepsilon_{4} \varepsilon_{2} \cdot p_{3}-v \cdot p_{23} \varepsilon_{2} \cdot \varepsilon_{4} \varepsilon_{3} \cdot p_{2}+v \cdot p_{3} \varepsilon_{2} \cdot \varepsilon_{3} \varepsilon_{4} \cdot p_{2}-v \cdot p_{2} \varepsilon_{2} \cdot \varepsilon_{3} \varepsilon_{4} \cdot p_{3}\right)\right) \\
& +\frac{1}{s_{34} s_{234}}\left(\varepsilon_{2} \cdot p_{34}\left(\varepsilon_{3} \cdot p_{4} v \cdot \varepsilon_{4}-\varepsilon_{4} \cdot p_{3} v \cdot \varepsilon_{3}\right)+\varepsilon_{2} \cdot \varepsilon_{4} v \cdot p_{2} \varepsilon_{3} \cdot p_{4}-\varepsilon_{4} \cdot p_{2} \varepsilon_{3} \cdot p_{4} v \cdot \varepsilon_{2}\right. \\
& +\varepsilon_{3} \cdot \varepsilon_{4} v \cdot p_{3} \varepsilon_{2} \cdot p_{34}+\varepsilon_{3} \cdot \varepsilon_{4} v \cdot p_{2} \varepsilon_{2} \cdot p_{3}-\varepsilon_{2} \cdot \varepsilon_{3} v \cdot p_{2} \varepsilon_{4} \cdot p_{3}+\varepsilon_{3} \cdot p_{2} \varepsilon_{4} \cdot p_{3} v \cdot \varepsilon_{2} \\
& \left.\left.-\frac{1}{4} s_{23} \varepsilon_{3} \cdot \varepsilon_{4} v \cdot \varepsilon_{2}+\frac{1}{4} s_{24} \varepsilon_{3} \cdot \varepsilon_{4} v \cdot \varepsilon_{2}-\frac{1}{4} s_{34} \varepsilon_{2} \cdot \varepsilon_{3} v \cdot \varepsilon_{4}+\frac{1}{4} s_{34} \varepsilon_{2} \cdot \varepsilon_{4} v \cdot \varepsilon_{3}\right)\right] \text {, }
\end{aligned}
$$

where

$$
p_{i_{1} i_{2} \cdots i_{r}}:=p_{i_{1}}+p_{i_{2}}+\cdots+p_{i_{r}}, \quad s_{i_{1} i_{2} \cdots i_{r}}:=p_{i_{1} i_{2} \cdots i_{r}}^{2} .
$$

Note that the gluon-quark amplitude in HEFT does not depend on the soft momentum of the heavy particle. One can also check that the leading order term in $1 / m$ of the full five-point gluon-quark amplitude is identical to (2.7).

\section{Traditional double copy construction}

In the previous section we reviewed an example of a heavy-particle effective theory and presented a few tree-level amplitudes derived from the leading-order Feynman rules. We chose to work with fermions as heavy particles, however it is important to note that the results thus obtained are in fact independent of the spin of the heavy matter fields [8]. The corresponding effective theory for gravity, studied using a form of the colour-kinematics duality and double copy of HEFT in [80], also exhibits universal behaviour at leading order in the heavy-mass limit, which is related to the universal coupling between matter and gravitons [82, 83].

Next, we discuss quark-graviton amplitudes for the full theory as computed using the traditional form of the colour-kinematics duality. The leading-order parts of these amplitudes in the heavy-mass limit can then be obtained by an expansion in the (inverse) heavy mass. ${ }^{3}$ In this form of the double copy one sums over all cubic graphs, and the numerators

\footnotetext{
${ }^{3}$ The quark-graviton amplitudes can also be obtained from Feynman rules [84, 85].
} 
are in general not gauge invariant. The double copy of QCD with massive fermions was studied in [77, 80], while the double copy of massive scalar QCD was investigated in [79].

The BCJ numerators for the three-, four- and five-point amplitudes can be cast in the form $[27,62,77]$

$$
\begin{aligned}
N_{3}(123) & =\bar{Q}_{2} Q, \\
N_{4}(1234) & =\bar{Q} \ddagger_{2}\left(\not p_{12}+m\right) \ddagger_{3} Q, \\
N_{5}(12345) & =\bar{Q}_{2}\left(\not \not_{12}+m\right) \ddagger_{3}\left(\not \not_{123}+m\right) \ddagger_{4} Q+\frac{2}{3}\left(p_{123}^{2}-m^{2}\right)\left(\bar{Q}_{2} \ddagger_{3} \ddagger_{4} Q-\varepsilon_{3} \cdot \varepsilon_{4} \bar{Q}_{2} Q\right) .
\end{aligned}
$$

The three-point graviton amplitude is obtained by just squaring the BCJ numerator

$$
A_{3}^{\mathrm{GR}-\mathrm{Q}}=\left[N_{3}(123)\right]^{2} .
$$

The four-point amplitude is obtained by summing over the following three cubic graphs with two gravitons and two fermions:

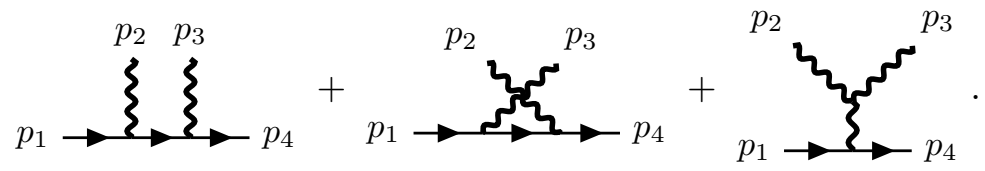

The numerator of each graph is the square of the corresponding BCJ numerator. The four-point amplitude is then

$$
A_{4}^{\mathrm{GR}-\mathrm{Q}}=\frac{N_{4}(1234)^{2}}{2 p_{1} \cdot p_{2}}+\frac{N_{4}(1324)^{2}}{2 p_{1} \cdot p_{3}}+\frac{N_{4}(1[2,3] 4)^{2}}{s_{23}}
$$

where $N_{4}(1324)$ is obtained from $N_{4}(1234)$ by swapping the indices 2,3 and the bracket on the external labels denotes the commutator of the indices, e.g.

$$
N_{4}(1[2,3] 4):=N_{4}(1234)-N_{4}(1324)
$$

The four-point amplitude is manifestly invariant under the exchange of particles 2 and 3 . Similarly the five-point amplitude is obtained by summing over all cubic graphs with two scalars and three external gravitons:
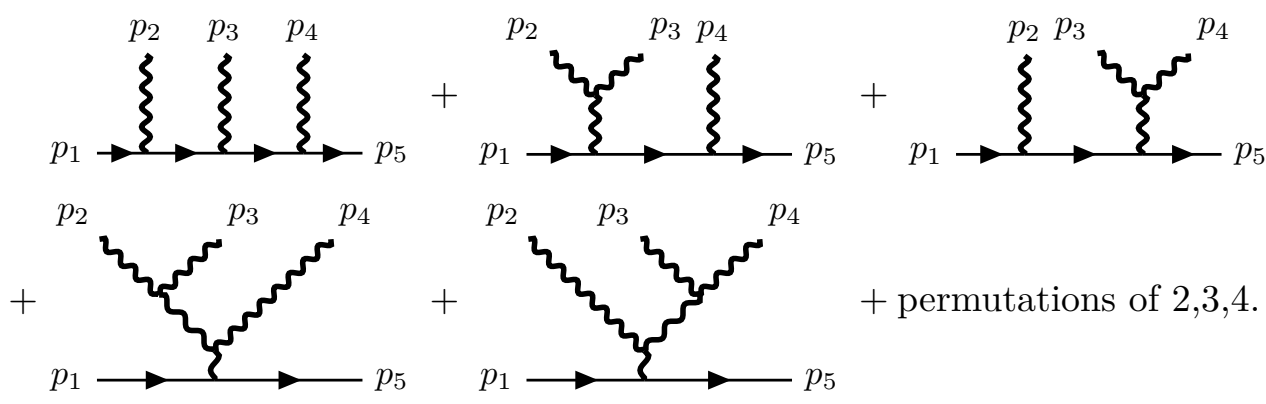

+ permutations of $2,3,4$. 
Computing the above diagrams, one gets

$$
\begin{aligned}
A_{5}^{\mathrm{GR}-\mathrm{Q}}= & \frac{N_{5}(12345)^{2}}{2 p_{1} \cdot p_{2}\left(2 p_{1} \cdot p_{23}+s_{23}\right)}+\frac{N_{5}(1[2,3] 45)^{2}}{2 s_{23}\left(2 p_{1} \cdot p_{23}+s_{23}\right)}+\frac{N_{5}(12[3,4] 5)^{2}}{4 s_{34} p_{1} \cdot p_{2}} \\
& +\frac{N_{5}(1[2,[3,4]] 5)^{2}}{2 s_{34} s_{234}}+\text { permutations of } 2,3,4 .
\end{aligned}
$$

The leading-order term in the heavy-mass expansion is obtained by simply retaining the $\mathcal{O}\left(m^{2}\right)$ terms in (3.7), and we have checked that it agrees with the same quantity as computed with the KLT duality.

A few comments are in order here. First, we note that in this traditional form of the double copy one has to sum over all possible cubic graphs; furthermore, gauge invariance of the amplitudes is not manifest due to the explicit appearance of polarisation vectors in the numerators $N_{i}$. Inspired by recent work on the kinematic algebra of BCJ numerators [62], in the next section we will introduce an improved version of the double copy, which will lead to much more compact expressions for the amplitudes. These will be used in [81] for the computation of classical quantities in gravity at loop level.

\section{A novel double copy from gauge-invariant BCJ numerators}

We now present our alternative form of the colour-kinematics duality for the gluon-matter amplitudes directly at leading order in the HEFT, avoiding the need to perform a heavymass expansion. This double copy is motivated by the work of one of the present authors [62] on the algebraic structure of numerators that are consistent with the colourkinematics duality, and allows us to generate the BCJ numerators directly. As we will see, this construction has several advantages:

1. The new BCJ numerators are automatically gauge invariant and unique.

2. Only a subset of the usual cubic diagrams contributes.

3. As a consequence, we obtain much more compact expressions for the amplitudes than those derived from the traditional BCJ construction discussed in the previous section.

We have tested this new method by explicitly constructing numerators up to six particles at tree level, although in principle the method applies to $n$-particle numerators.

\subsection{Background for the colour-kinematics algebra from fusion rules}

We now describe the new construction of BCJ numerators, which is based on the fusion product between two heavy-mass currents. Following the notation of [62] these currents are denoted by $J_{a_{1} \otimes a_{2} \otimes \cdots \otimes a_{r}},{ }^{4}$ and their labels $a_{i}^{\mu}$ can either be momenta or polarisation vectors. Since $\bar{Q} \phi_{i} Q \sim m v \cdot a_{i}$ in the HEFT, we define

$$
J_{a}=m v \cdot a .
$$

\footnotetext{
${ }^{4}$ The notation for these currents is inspired by the form of tensor currents in QCD, $\bar{Q} \phi_{1} \phi_{2} \cdots \phi_{r} Q$.
} 
We also require that the tensor currents $J_{a_{1} \otimes a_{2} \cdots a_{i} \otimes a_{j} \cdots \otimes a_{r}}$ satisfy the Clifford algebra

$$
J_{a_{1} \otimes a_{2} \cdots a_{i} \otimes a_{j} \cdots \otimes a_{r}}=-J_{a_{1} \otimes a_{2} \cdots a_{j} \otimes a_{i} \cdots \otimes a_{r}}+2 a_{i} \cdot a_{j} J_{a_{1} \otimes a_{2} \cdots \hat{a}_{i} \otimes \hat{a}_{j} \cdots \otimes a_{r}},
$$

for each component. This property is inherited from the QCD currents.

The on-shell condition for the external quarks also gives the relations

$$
\begin{aligned}
J_{a_{1} \otimes a_{2} \otimes \cdots a_{n} \otimes p_{12 \ldots n-1}} & =m J_{a_{1} \otimes a_{2} \otimes \cdots \otimes a_{n}}, \\
J_{p_{1} \otimes a_{1} \otimes a_{2} \otimes \cdots \otimes a_{n}} & =m J_{a_{1} \otimes a_{2} \otimes \cdots \otimes a_{n}},
\end{aligned}
$$

and the currents satisfy a bilinear fusion rule of the form

$$
J_{X} \star J_{Y}=\sum_{Z} F_{X Y}^{Z} J_{Z}
$$

For instance [62]

$$
J_{\varepsilon_{2}} \star J_{\varepsilon_{3}}:=2\left(\frac{s_{23}}{4} \frac{v \cdot \varepsilon_{3}}{v \cdot p_{2}} J_{\varepsilon_{2}}-\frac{1}{2} J_{\varepsilon_{2} \otimes \varepsilon_{3} \otimes p_{2}}+\varepsilon_{3} \cdot p_{2} J_{\varepsilon_{2}}\right) .
$$

The fusion rule always generates a rational function which we call the pre-numerator:

$$
\mathcal{N}_{n}(23 \cdots n-1, v):=\left.{ }^{2}\right|^{3 \cdots n-1}=J_{\varepsilon_{2}} \star J_{\varepsilon_{3}} \star \cdots \star J_{\varepsilon_{n-1}},
$$

where we always assume associativity of the $\star$-product. In this diagram, the red box denotes the two massive particles, while the lines correspond to the massless particles (gluons or gravitons). The general form of the fusion rule with these properties is known in pure Yang-Mills from [62] for $n$-point MHV amplitudes and for NMHV amplitudes up to eight points. The claim is that BCJ numerators in HEFT can be written efficiently in terms of the pre-numerator, in a way we describe below.

To begin with, it is useful to introduce the notion of ordered and un-ordered nested commutators. In the case of ordered nested commutators, which is relevant for colourordered amplitudes, the order of a set of indices is fixed while commutators are applied in all possible ways. For example, for $n=5$, the ordered set $\{2,3,4\}$ gives rise to two ordered, nested commutators: $[[2,3], 4]$ and $[2,[3,4]]$. In the case of graviton amplitudes, we need to include also un-ordered nested commutators (omitting numerators that differ by minus signs): $[[2,3], 4],[[2,4], 3]$ and $[[3,4], 2]$. Then the gluon-matter and graviton-matter amplitudes are given by the following expressions:

$$
\begin{aligned}
& A_{n}^{\mathrm{YM}-\mathrm{M}}(12 \cdots n)=\sum_{\Gamma \in \text { ordered commutators }\{2,3, \cdots, n-1\}} \frac{\mathcal{N}_{n}(\Gamma, v)}{d_{\Gamma}}, \\
& \sum_{\Gamma \in \text { non-ordered commutators }\{2,3, \cdots, n-1\}}^{\mathrm{GR}-\mathrm{M}}(12 \cdots n)=\sum_{\left.\Gamma, 1 \mathcal{N}_{n}(\Gamma, v)\right]^{2}} d_{\Gamma}
\end{aligned}
$$

where particles 1 and $n$ are heavy and all others are massless. Each nested commutator (and hence BCJ numerator) is in one-to-one correspondence with a specific cubic graph, 
from which one can also read off the relevant massless scalar propagators, denoted as $d_{\Gamma}$ in (4.7). For instance, the nested commutator [[2,3],4] and the associated BCJ numerator corresponds to the cubic graph

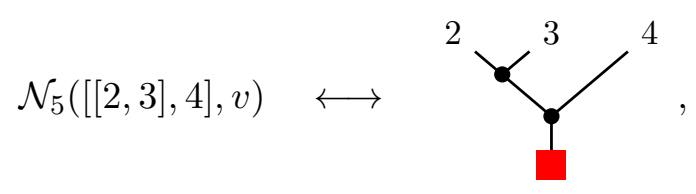

where in this case $d_{[[2,3], 4]}=s_{23} s_{234}$. We also note an important feature of this BCJ representation, namely that we only sum over cubic graphs corresponding to nested commutators. In particular, only massless propagators appear within the cubic graph - the two massive particles always connect to the graph via a single cubic vertex.

Each BCJ numerator $\mathcal{N}_{n}(\Gamma, v)$ can be conveniently obtained by acting with specific operators on the pre-numerator. For instance, a left-nested commutator is written as

$$
\mathcal{N}_{n}([\ldots[[2,3], 4], \ldots, n-1], v):=\mathbb{L}(2,3,4, \ldots, n-1) \circ \mathcal{N}_{n}(234 \ldots n-1, v),
$$

where $\mathbb{L}(2,3,4, \ldots, n-1)$ is the $\mathbb{L}$-operator introduced in [63] as a group algebra element [86, 87] and in [88] as a free Lie algebra element,

$$
\mathbb{L}\left(i_{1}, i_{2}, \ldots, i_{r}\right):=\left[\mathbb{I}-\mathbb{P}_{\left(i_{2} i_{1}\right)}\right]\left[\mathbb{I}-\mathbb{P}_{\left(i_{3} i_{2} i_{1}\right)}\right] \cdots\left[\mathbb{I}-\mathbb{P}_{\left(i_{r} \ldots i_{2} i_{1}\right)}\right]
$$

Here $\mathbb{P}_{\left(j_{1} j_{2} j_{3} \ldots j_{m}\right)}$ denotes the cyclic permutation $j_{1} \rightarrow j_{2} \rightarrow j_{3} \rightarrow \cdots \rightarrow j_{m} \rightarrow j_{1}$. For instance

$$
\begin{aligned}
\mathbb{I} \circ \mathcal{N}_{6}(2345, v) & =\mathcal{N}_{6}(2345, v), \\
\mathbb{P}_{(432)} \circ \mathcal{N}_{6}(2345, v) & =\mathcal{N}_{6}(4235, v) .
\end{aligned}
$$

Importantly, as we will discuss in more detail later, for each choice of $\Gamma$ the numerator $\mathcal{N}_{n}(\Gamma, v)$ is consistent with colour-kinematics duality.

We have successfully checked this conjecture up to six points. An important observation is that the numerators we obtain in this approach are all gauge invariant, as will become clear in concrete examples presented later. The general method to determine the fusion rules will be described elsewhere, while in this paper we will focus on describing a method to construct the pre-numerator from an ansatz, and from this, the BCJ numerators $\mathcal{N}_{n}(\Gamma, v)$.

\subsection{General method to construct the pre-numerator}

Since the fusion rule introduced in (4.4) is not yet known in general, our strategy consists in writing down a general ansatz for the pre-numerator, in terms of which the BCJ numerators $\mathcal{N}_{n}(\Gamma, v)$ are written. We then require that these BCJ numerators $\mathcal{N}_{n}(\Gamma, v)$ generate the correct amplitudes when inserted in (4.7). In all cases we have considered, this procedure leads to a unique, gauge-invariant answer for the BCJ numerators (while in general the pre-numerator has undetermined coefficients). 
The building blocks of the ansatz are products of the following Lorentz-invariant quantities: ${ }^{5}$

$$
\frac{1}{v \cdot p_{i_{1} \ldots i_{r}}}, \quad \varepsilon_{i} \cdot \varepsilon_{j}, \quad \varepsilon_{i} \cdot p_{j} \quad v \cdot \varepsilon_{i}, \quad v \cdot p_{i}, \quad s_{i j}, \quad J_{\varepsilon_{i}}, \quad J_{p_{i}}
$$

In fact, in constructing the ansatz we can restrict the vector currents $J$ to only those involving $J_{p_{2}}$ and $J_{\varepsilon_{2}}$, since the others can be generated with the $\mathbb{L}(2,3,4, \ldots, n-1)$ operator.

There are several constraints to impose on our ansatz. First, for an $n$-point YangMills amplitude, there are at most $n-3$ propagators of the heavy particles and no double poles. Second, it follows from the Feynman rules (2.4) that the overall scaling degree in $v$ should be one. Third, we have observed that it is sufficient to restrict the power of $s_{i j}$ in a particular term in the ansatz to be the same as the total number of massive propagators in that term. Hence a general term in the ansatz for the pre-numerator $\mathcal{N}^{(\text {ans })}(234 \cdots n-1, v)$ has the form

$$
\left(\prod_{h=1}^{d} \frac{1}{v \cdot p_{i_{1}^{(h)} \ldots i_{r_{h}}^{(h)}}}\right)\left(\prod_{h=1}^{d} s_{i^{(h)} j^{(h)}}\right)\left(\prod_{h=1}^{d} v \cdot a_{i^{(h)}}\right)\left(\prod_{h=1}^{d^{\prime}} \varepsilon_{i^{(h)}} \cdot a_{j^{(h)}}\right) J_{a_{2}},
$$

where $d \in[1, n-3]$, and $a$ denotes either $\varepsilon$ or $p$. Finally, the ansatz is further constrained by power counting and multi-linearity with respect to external polarisation vectors (which fixes $\left.d^{\prime}\right)$.

An important comment is in order here. The terms without any denominator are fixed by the MHV sector in the pure Yang-Mills theory ${ }^{6}$ with $\epsilon_{1}$ replaced by $m v$. In fact, we have determined the MHV fusion rules, and hence the Yang-Mills pre-numerator for this sector, which has the following form:

$$
\begin{aligned}
\mathcal{N}_{n}^{(0)}(234 \cdots n-1, v) & =2^{n-3}\left(\left(\prod_{j=3}^{n-1} \varepsilon_{j} \cdot p_{2 \ldots j}\right) J_{\varepsilon_{2}}\right. \\
& \left.-\frac{1}{2} \sum_{h>\ell=2}^{n-1}(-1)^{\ell}\left(\prod_{j=\ell+1, j \neq h}^{n-1} \varepsilon_{j} \cdot p_{2 \ldots j}\right) \varepsilon_{2} \cdot H_{3} \cdot H_{4} \cdots H_{\ell-1} \cdot p_{1 \ldots \ell} J_{\varepsilon_{\ell} \otimes \varepsilon_{h} \otimes p_{2}}\right),
\end{aligned}
$$

where $H_{i}^{\mu \nu}=p_{2 \ldots i}^{\mu} \varepsilon_{i}^{\nu}-\eta^{\mu \nu} \varepsilon_{i} \cdot p_{2 \ldots i}$. Furthermore, when $\ell=3$ in the sum in the second line of (4.14), the product of $H$ factors should be replaced by $\eta_{\mu \nu}$, while when $\ell=2$, $\varepsilon_{2} \cdot H_{3} \cdot H_{4} \cdots H_{\ell-1} \cdot p_{1 \ldots \ell}$ is replaced by 1 .

We will find it convenient to write the ansatz for the $n$-particle pre-numerator as the sum of the purely polynomial MHV part and a yet to be determined remainder containing massive propagators:

$$
\mathcal{N}_{n}(234 \cdots n-1, v):=\mathcal{N}_{n}^{(0)}(234 \cdots n-1, v)+\mathcal{N}_{n}^{(\text {ans })}(234 \cdots n-1, v) .
$$

\footnotetext{
${ }^{5}$ Although $J_{a}$ is proportional to $v \cdot a$, we prefer to treat these two quantities as separate as their origin in the QCD amplitude is different, specifically the $J_{a}$ terms arise from fermion bilinears while the $v \cdot a$ terms from expanding propagators. Note that in constructing the ansatz, we always fix $J_{a}$ to be either $J_{p_{2}}$ or $J_{\varepsilon_{2}}$ from the requirement of full crossing symmetry with respect to the gravitons.

${ }^{6}$ This is further discussed in section 6 .
} 
In the next section we will apply this general procedure to construct BCJ numerators in explicit examples.

\section{Applications of the new double copy}

We now give examples of the construction of gauge-invariant BCJ numerators up to sixpoint amplitudes. We remind the reader that in what follows $p_{1}=m v \simeq-p_{n}$ always denote the momenta of the hard particles.

\subsection{Three-point numerator}

At three points, the pre-numerator is simply

$$
\mathcal{N}_{3}(2, v):=\stackrel{2}{\mid}=J_{\varepsilon_{2}}=m v \cdot \varepsilon_{2},
$$

and the amplitudes with a gluon or a graviton are

$$
A_{3}^{\mathrm{YM}-\mathrm{M}}(123)=\mathcal{N}_{3}(2, v), \quad A_{3}^{\mathrm{GR}-\mathrm{M}}(123)=\left[\mathcal{N}_{3}(2, v)\right]^{2} .
$$

\subsection{Four-point numerator}

At four points, we know from (4.5) that the pre-numerator is

$$
\mathcal{N}_{4}(23, v):={ }^{2} \searrow /^{3}=2\left(\frac{s_{23} v \cdot \varepsilon_{3}}{4 v \cdot p_{2}} J_{\varepsilon_{2}}-\frac{1}{2} J_{\varepsilon_{2} \otimes \varepsilon_{3} \otimes p_{2}}+\varepsilon_{3} \cdot p_{2} J_{\varepsilon_{2}}\right) .
$$

Then the amplitudes with two gluons or two gravitons are

$$
A_{4}^{\mathrm{YM}-\mathrm{M}}=\frac{\mathcal{N}_{4}([2,3], v)}{s_{23}}, \quad A_{4}^{\mathrm{GR}-\mathrm{M}}=\frac{\left[\mathcal{N}_{4}([2,3], v)\right]^{2}}{s_{23}},
$$

where

$$
\mathcal{N}_{4}([2,3], v):={ }^{3}:=\mathbb{L}(2,3) \circ \mathcal{N}_{4}(23, v)=2 m\left(\frac{v \cdot F_{2} \cdot F_{3} \cdot v}{v \cdot p_{3}}\right)
$$

Here $F_{i}^{\mu \nu}=p_{i}^{\mu} \varepsilon_{i}^{\nu}-\varepsilon_{i}^{\mu} p_{i}^{\nu}$, and we have used the fact that $J_{a}=m v \cdot a$ for a vector current. Note that terms containing tensor currents always cancel out among themselves after using the Clifford algebra. For instance, the current $J_{\varepsilon_{2} \otimes \varepsilon_{3} \otimes p_{2}}$ appears in the form of a commutator, which we can recast as

$$
\begin{aligned}
J_{\varepsilon_{2} \otimes \varepsilon_{3} \otimes p_{2}}-J_{\varepsilon_{3} \otimes \varepsilon_{2} \otimes p_{3}} & =2 \varepsilon_{2} \cdot \varepsilon_{3} J_{p_{2}}-J_{\varepsilon_{3} \otimes \varepsilon_{2} \otimes p_{23}}=2 \varepsilon_{2} \cdot \varepsilon_{3} J_{p_{2}}-J_{\varepsilon_{3} \otimes \varepsilon_{2} \otimes p_{123}}+J_{\varepsilon_{3} \otimes \varepsilon_{2} \otimes p_{1}} \\
& =2 \varepsilon_{2} \cdot \varepsilon_{3} J_{p_{2}}+2 \varepsilon_{2} \cdot p_{1} J_{\varepsilon_{3}}-2 p_{1} \cdot \varepsilon_{3} J_{\varepsilon_{2}}-J_{\varepsilon_{3} \otimes \varepsilon_{2} \otimes p_{123}}+J_{p_{1} \otimes \varepsilon_{3} \otimes \varepsilon_{2}} \\
& =2 \varepsilon_{2} \cdot \varepsilon_{3} J_{p_{2}}+2 \varepsilon_{2} \cdot p_{1} J_{\varepsilon_{3}}-2 p_{1} \cdot \varepsilon_{3} J_{\varepsilon_{2}}+m J_{\varepsilon_{3} \otimes \varepsilon_{2}}-m J_{\varepsilon_{3} \otimes \varepsilon_{2}} \\
& =2 \varepsilon_{2} \cdot \varepsilon_{3} J_{p_{2}}+2 \varepsilon_{2} \cdot p_{1} J_{\varepsilon_{3}}-2 p_{1} \cdot \varepsilon_{3} J_{\varepsilon_{2}}
\end{aligned}
$$

where we have repeatedly used (4.2) and the on-shell conditions (4.3). Note the absence of tensor currents in the last line of (5.6). We also observe that (5.5) is expressed in terms of gauge-invariant quantities only. 


\subsection{Five-point numerator}

From five points on, we need to use the method described in section 4.2 to determine the BCJ numerators. According to (4.14), the part of the pre-numerator without linear propagators, that is $\mathcal{N}_{5}^{(0)}(234, v)$, is given by

$$
\begin{aligned}
\mathcal{N}_{5}^{(0)}(234, v)= & 4\left(p_{2} \cdot \varepsilon_{3} p_{23} \cdot \varepsilon_{4} J_{\varepsilon_{2}}\right. \\
& \left.-\frac{1}{2} p_{23} \cdot \varepsilon_{4} J_{\varepsilon_{2} \otimes \varepsilon_{3} \otimes p_{2}}-\frac{1}{2} p_{23} \cdot \varepsilon_{3} J_{\varepsilon_{2} \otimes \varepsilon_{4} \otimes p_{2}}+\frac{1}{2} p_{23} \cdot \varepsilon_{2} J_{\varepsilon_{3} \otimes \varepsilon_{4} \otimes p_{2}}\right) .
\end{aligned}
$$

We now write the ansatz for the remaining terms in the pre-numerator, as per (4.15). We divide these terms into three sectors of the form

$$
v \cdot \varepsilon v \cdot \varepsilon, \quad \quad \varepsilon \cdot \varepsilon v \cdot \varepsilon, \quad v \cdot \varepsilon v \cdot \varepsilon p \cdot \varepsilon .
$$

The most general ansatz made of terms of this form contains 42 parameters, denoted by $x_{i, j}$ below:

$$
\begin{aligned}
\mathcal{N}_{5}^{\left(\text {ans }_{1}\right)}(234, v)= & \left(\frac{x_{1,1} s_{23}^{2} v \cdot \epsilon_{3} v \cdot \epsilon_{4}}{v \cdot p_{2} v \cdot p_{23}}+\frac{x_{1,2} s_{24} s_{23} v \cdot \epsilon_{3} v \cdot \epsilon_{4}}{v \cdot p_{2} v \cdot p_{23}}+\frac{x_{1,3} s_{24}^{2} v \cdot \epsilon_{3} v \cdot \epsilon_{4}}{v \cdot p_{2} v \cdot p_{23}}\right. \\
& \left.+\frac{x_{1,4} s_{34} s_{23} v \cdot \epsilon_{3} v \cdot \epsilon_{4}}{v \cdot p_{2} v \cdot p_{23}}+\frac{x_{1,5} s_{24} s_{34} v \cdot \epsilon_{3} v \cdot \epsilon_{4}}{v \cdot p_{2} v \cdot p_{23}}+\frac{x_{1,6} s_{34}^{2} v \cdot \epsilon_{3} v \cdot \epsilon_{4}}{v \cdot p_{2} v \cdot p_{23}}\right) J_{\epsilon_{2}}, \\
\mathcal{N}_{5}^{\left(\text {ans }_{2}\right)}(234, v)= & \left(\frac{x_{2,1} s_{23} \epsilon_{3} \cdot \epsilon_{4} v \cdot p_{3}}{v \cdot p_{2}}+11 \text { terms }\right) J_{\epsilon_{2}} \\
\mathcal{N}_{5}^{(\text {ans })}(234, v)= & \left(\frac{x_{3,1} s_{23} \epsilon_{4} \cdot p_{3} v \cdot \epsilon_{3}}{v \cdot p_{2}}+23 \text { terms }\right) J_{\epsilon_{2}} .
\end{aligned}
$$

We solve for the parameters according to (4.7), and arrive at a pre-numerator with 11 undetermined parameters:

$$
\begin{aligned}
\mathcal{N}_{5}(234, v):= & \searrow^{3} /\left.\right|^{4} \\
= & 4\left(\frac{s_{23} \epsilon_{4} \cdot p_{3} v \cdot \epsilon_{3}}{2 v \cdot p_{2}}+\frac{s_{34} \epsilon_{3} \cdot p_{2} v \cdot \epsilon_{4}}{2 v \cdot p_{23}}+\frac{s_{23} \epsilon_{3} \cdot \epsilon_{4} v \cdot p_{4}}{2 v \cdot p_{2}}+\frac{s_{23} s_{34} v \cdot \epsilon_{3} v \cdot \epsilon_{4}}{8 v \cdot p_{2} v \cdot p_{23}}\right) J_{\epsilon_{2}} \\
& +\mathcal{N}_{5}^{(0)}(234, v)+x_{1,1}\left(\frac{s_{23}^{2} v \cdot \epsilon_{3} v \cdot \epsilon_{4}}{v \cdot p_{2} v \cdot p_{23}}-\frac{s_{34}^{2} v \cdot \epsilon_{3} v \cdot \epsilon_{4}}{v \cdot p_{2} v \cdot p_{23}}\right) J_{\epsilon_{2}}+\cdots,
\end{aligned}
$$

where we have indicated for brevity only one of the terms with a free parameter. Remarkably, we find that the terms with undetermined parameters vanish under the action of $\mathbb{L}(2,3,4)$ :

$$
\mathbb{L}(2,3,4) \circ\left[x_{1,1}\left(\frac{s_{23}^{2} v \cdot \epsilon_{3} v \cdot \epsilon_{4}}{v \cdot p_{2} v \cdot p_{23}}-\frac{s_{34}^{2} v \cdot \epsilon_{3} v \cdot \epsilon_{4}}{v \cdot p_{2} v \cdot p_{23}}\right) J_{\epsilon_{2}}+\cdots\right]=0 .
$$


As a consequence, and in contradistinction with the expectation from pure Yang-Mills amplitudes, we arrive at a unique solution for the BCJ numerator.

Summarising, the BCJ numerator for the left-nested commutator is

$$
\mathcal{N}_{5}([[2,3], 4], v):=\overbrace{}^{4}:=\mathbb{L}(2,3,4) \circ \mathcal{N}_{5}(234, v) \text {. }
$$

Importantly, we find that this BCJ numerator can be rewritten in a manifestly gaugeinvariant form as

$$
\mathcal{N}_{5}([[2,3], 4], v)=\mathbb{L}(2,3,4) \circ\left[4 m \frac{v \cdot F_{2} \cdot F_{3} \cdot V_{3} \cdot F_{4} \cdot v}{v \cdot p_{3} v \cdot p_{4}}\right],
$$

where $V_{i}^{\mu \nu}=v^{\mu} p_{i}^{\nu}$. Then the colour-ordered amplitude in the novel colour-kinematics duality form is

$$
A_{5}^{\mathrm{YM}-\mathrm{M}}(12345)=\frac{\mathcal{N}_{5}([[2,3], 4], v)}{s_{234} s_{23}}+\frac{\mathcal{N}_{5}([2,[3,4]], v)}{s_{234} s_{34}} .
$$

Note that these two terms are related by exchanging $2 \leftrightarrow 4$. By the double copy, the gravity amplitude is obtained immediately as

$$
A_{5}^{\mathrm{GR}-\mathrm{M}}(12345)=\frac{\left[\mathcal{N}_{5}([[2,3], 4], v)\right]^{2}}{s_{234} s_{23}}+\frac{\left[\mathcal{N}_{5}([[2,4], 3], v)\right]^{2}}{s_{234} s_{24}}+\frac{\left[\mathcal{N}_{5}([[3,4], 2], v)\right]^{2}}{s_{234} s_{34}} .
$$

We have checked that (5.15) agrees with the $\mathcal{O}\left(\mathrm{m}^{2}\right)$ term of (3.7). It is useful to pause and contrast these two expressions to appreciate the simplicity of our approach. First, (5.15) is much more compact than (3.7); furthermore, expanding (3.7) to $\mathcal{O}\left(\mathrm{m}^{2}\right)$ gives rise to a very large number of terms lacking any particular structure. The compactness, and manifest gauge invariance and locality of our HEFT amplitudes are of considerable advantage when these expressions are fed into unitarity cuts.

We also comment that the numerators we have constructed automatically satisfy the Jacobi relations. For instance, by definition we have

$$
\begin{aligned}
& \mathcal{N}_{5}([[2,3], 4], v)=\mathcal{N}_{5}(234, v)-\mathcal{N}_{5}(324, v)-\mathcal{N}_{5}(423, v)+\mathcal{N}_{5}(432, v) \\
& \mathcal{N}_{5}([[2,4], 3], v)=\mathcal{N}_{5}(243, v)-\mathcal{N}_{5}(423, v)-\mathcal{N}_{5}(324, v)+\mathcal{N}_{5}(342, v) \\
& \mathcal{N}_{5}([2,[3,4]], v)=\mathcal{N}_{5}(234, v)-\mathcal{N}_{5}(243, v)-\mathcal{N}_{5}(342, v)+\mathcal{N}_{5}(432, v),
\end{aligned}
$$

which makes the Jacobi relation manifest,

$$
\mathcal{N}_{5}([2,[3,4]], v)=\mathcal{N}_{5}([[2,3], 4], v)-\mathcal{N}_{5}([[2,4], 3], v)
$$

Finally, note that one can construct $\mathcal{N}_{5}([[2,4], 3], v)$ from $\mathcal{N}_{5}([[2,3], 4], v)$ by simply swapping labels 3 and 4 . 


\subsection{Six-point numerator}

Similarly to what was done at five points, using again (4.14) we find that, at six points, the polynomial part of the pre-numerator, $\mathcal{N}_{6}^{(0)}(2345, v)$, has the form

$$
\begin{aligned}
& \mathcal{N}_{6}^{(0)}(2345, v)=8\left(J_{\varepsilon_{2}} p_{2} \cdot \varepsilon_{3} p_{23} \cdot \varepsilon_{4} p_{234} \cdot \varepsilon_{5}\right. \\
& -\frac{1}{2} p_{23} \cdot \varepsilon_{4} p_{234} \cdot \varepsilon_{5} J_{\varepsilon_{2} \otimes \varepsilon_{3} \otimes p_{2}}-\frac{1}{2} p_{2} \cdot \varepsilon_{3} p_{234} \cdot \varepsilon_{5} J_{\varepsilon_{2} \otimes \varepsilon_{4} \otimes p_{2}}-\frac{1}{2} p_{2} \cdot \varepsilon_{3} p_{23} \cdot \varepsilon_{4} J_{\varepsilon_{2} \otimes \varepsilon_{5} \otimes p_{2}} \\
& \left.+\frac{1}{2} p_{3} \cdot \varepsilon_{2} p_{234} \cdot \varepsilon_{5} J_{\varepsilon_{3} \otimes \varepsilon_{4} \otimes p_{2}}+\frac{1}{2} p_{3} \cdot \varepsilon_{2} p_{23} \cdot \varepsilon_{4} J_{\varepsilon_{3} \otimes \varepsilon_{5} \otimes p_{2}}+\frac{1}{2}\left(\varepsilon_{2} \cdot p_{4} \varepsilon_{3} \cdot p_{2}-\varepsilon_{2} \cdot p_{3} \varepsilon_{3} \cdot p_{4}\right) J_{\varepsilon_{4} \otimes \varepsilon_{5} \otimes p_{2}}\right) .
\end{aligned}
$$

The most general ansatz for the remainder of the pre-numerator can be decomposed into six sectors. The number of free parameters and physical constraints arising from (4.7) are shown in the table below:

\begin{tabular}{|c|c|c|c|c|c|c|}
\hline sector & $v \cdot \varepsilon v \cdot \varepsilon v \cdot \varepsilon v \cdot \varepsilon$ & $v \cdot \varepsilon v \cdot \varepsilon v \cdot \varepsilon p \cdot \varepsilon$ & $v \cdot \varepsilon v \cdot \varepsilon \varepsilon \cdot \varepsilon$ & $v \cdot \varepsilon v \cdot \varepsilon p \cdot \varepsilon p \cdot \varepsilon$ & $v \cdot \varepsilon \varepsilon \cdot \varepsilon p \cdot \varepsilon$ & $\varepsilon \cdot \varepsilon \varepsilon \cdot \varepsilon$ \\
\hline \# of parameters & 280 & 1134 & 945 & 1128 & 1134 & 378 \\
\hline \# of constraints & 176 & 601 & 438 & 570 & 810 & 108 \\
\hline
\end{tabular}

Thus we arrive at a pre-numerator with 2296 free parameters:

$$
\begin{aligned}
& \mathcal{N}_{6}(2345, v):={ }^{2}>^{3} / /^{4}=8\left(\frac{s_{34} v \cdot \varepsilon_{3} v \cdot \varepsilon_{4} v \cdot \varepsilon_{5}}{8 v \cdot p_{2} v \cdot p_{23}}\left(\frac{s_{23} s_{45}}{2 v \cdot p_{234}}+\frac{s_{24} s_{45}}{3 v \cdot p_{234}}-\frac{s_{23} s_{35}}{3 v \cdot p_{4}}\right) J_{\varepsilon_{2}}\right. \\
& +\left(\frac{s_{23} s_{34} \varepsilon_{5} \cdot p_{4} v \cdot \varepsilon_{3} v \cdot \varepsilon_{4}}{4 v \cdot p_{2} v \cdot p_{23}}+\frac{s_{45} s_{34} \varepsilon_{3} \cdot p_{2} v \cdot \varepsilon_{4} v \cdot \varepsilon_{5}}{4 v \cdot p_{23} v \cdot p_{234}}-\frac{s_{23} s_{34} \varepsilon_{5} \cdot p_{3} v \cdot \varepsilon_{3} v \cdot \varepsilon_{4}}{8 v \cdot p_{2} v \cdot p_{4}}\right. \\
& \left.-\frac{s_{35} s_{34} \varepsilon_{3} \cdot p_{2} v \cdot \varepsilon_{4} v \cdot \varepsilon_{5}}{4 v \cdot p_{4} v \cdot p_{234}}-\frac{s_{23} s_{45} \varepsilon_{3} \cdot p_{4} v \cdot \varepsilon_{4} v \cdot \varepsilon_{5}}{4 v \cdot p_{2} v \cdot p_{234}}-\frac{s_{24} s_{45} \varepsilon_{3} \cdot p_{4} v \cdot \varepsilon_{4} v \cdot \varepsilon_{5}}{8 v \cdot p_{2} v \cdot p_{234}}\right) J_{\varepsilon_{2}} \\
& +\left(\frac{s_{23} s_{34} \varepsilon_{4} \cdot \varepsilon_{5} v \cdot p_{5} v \cdot \varepsilon_{3}}{4 v \cdot p_{2} v \cdot p_{23}}-\frac{s_{23} s_{34} \varepsilon_{3} \cdot \varepsilon_{5} v \cdot p_{5} v \cdot \varepsilon_{4}}{8 v \cdot p_{2} v \cdot p_{4}}+\frac{s_{23} s_{35} \varepsilon_{3} \cdot \varepsilon_{4} v \cdot p_{4} v \cdot \varepsilon_{5}}{8 v \cdot p_{23} v \cdot p_{234}}\right. \\
& \left.+\frac{s_{23} s_{45} \varepsilon_{3} \cdot \varepsilon_{4} v \cdot \varepsilon_{5}}{8 v \cdot p_{234}}\left(\frac{v \cdot p_{4}}{v \cdot p_{2}}+\frac{v \cdot p_{45}}{v \cdot p_{23}}\right)+\frac{s_{24} s_{45} \varepsilon_{3} \cdot \varepsilon_{4} v \cdot p_{4} v \cdot \varepsilon_{5}}{8 v \cdot p_{234}}\left(\frac{1}{v \cdot p_{2}}-\frac{1}{v \cdot p_{23}}\right)\right) J_{\varepsilon_{2}} \\
& +\left(\frac{\left(s_{23}-s_{45}\right) \varepsilon_{3} \cdot p_{4} \varepsilon_{5} \cdot p_{2} v \cdot \varepsilon_{4}}{2 v \cdot p_{34}}+s_{23} \varepsilon_{4} \cdot p_{3} v \cdot \varepsilon_{3}\left(\frac{\varepsilon_{5} \cdot p_{34}}{2 v \cdot p_{2}}-\frac{\varepsilon_{5} \cdot p_{2}}{2 v \cdot p_{34}}\right)+\frac{s_{25} \varepsilon_{3} \cdot p_{2} \varepsilon_{5} \cdot p_{4} v \cdot \varepsilon_{4}}{2 v \cdot p_{23}}\right. \\
& \left.+\frac{\left(s_{35}+s_{45}\right) \varepsilon_{3} \cdot p_{2} \varepsilon_{4} \cdot p_{23} v \cdot \varepsilon_{5}}{2 v \cdot p_{234}}-\frac{s_{34} \varepsilon_{3} \cdot p_{2} \varepsilon_{5} \cdot p_{23} v \cdot \varepsilon_{4}}{2 v \cdot p_{4}}-\frac{\left(s_{25}+s_{34}\right) \varepsilon_{3} \cdot p_{2} \varepsilon_{4} \cdot p_{5} v \cdot \varepsilon_{5}}{4 v \cdot p_{23}}\right) J_{\varepsilon_{2}} \\
& +\left(\frac{s_{23} \varepsilon_{3} \cdot \varepsilon_{5} v \cdot p_{5} \varepsilon_{4} \cdot p_{2}}{2 v \cdot p_{24}}+\frac{s_{23} \varepsilon_{3} \cdot \varepsilon_{5} v \cdot p_{5} \varepsilon_{4} \cdot p_{3}}{2 v \cdot p_{2}}+\frac{s_{23} \varepsilon_{3} \cdot \varepsilon_{4} v \cdot p_{4} \varepsilon_{5} \cdot p_{3}}{2 v \cdot p_{2}}+\frac{s_{23} \varepsilon_{3} \cdot \varepsilon_{4} v \cdot p_{45} \varepsilon_{5} \cdot p_{4}}{2 v \cdot p_{2}}\right. \\
& \left.-\frac{s_{23} \varepsilon_{4} \cdot \varepsilon_{5} v \cdot p_{5} \varepsilon_{3} \cdot p_{4}}{2 v \cdot p_{2}}-\frac{s_{23} \varepsilon_{3} \cdot \varepsilon_{4} v \cdot p_{4} \varepsilon_{5} \cdot p_{2}}{2 v \cdot p_{34}}+\frac{\left(s_{24}+s_{34}\right) \varepsilon_{4} \cdot \varepsilon_{5} v \cdot p_{5} \varepsilon_{3} \cdot p_{2}}{2 v \cdot p_{23}}\right) J_{\varepsilon_{2}} \\
& +\left(\frac{s_{25} \varepsilon_{2} \cdot \varepsilon_{3} \varepsilon_{4} \cdot \varepsilon_{5} v \cdot p_{4}}{4 v \cdot p_{23}}+\frac{s_{35} \varepsilon_{2} \cdot \varepsilon_{3} \varepsilon_{4} \cdot \varepsilon_{5} v \cdot p_{5}}{4 v \cdot p_{234}}+\frac{s_{45} \varepsilon_{2} \cdot \varepsilon_{4} \varepsilon_{3} \cdot \varepsilon_{5} v \cdot p_{5}}{4 v \cdot p_{234}}\right. \\
& \left.\left.-s_{45} \varepsilon_{2} \cdot \varepsilon_{5} \varepsilon_{3} \cdot \varepsilon_{4}\left(\frac{v \cdot p_{4}}{2 v \cdot p_{34}}+\frac{v \cdot p_{5}}{4 v \cdot p_{234}}\right)\right) J_{p_{2}}\right)+\mathcal{N}_{6}^{(0)}(2345, v)+\cdots,
\end{aligned}
$$


where we omit all the terms with free parameters. Then the BCJ numerator from the left-nested commutator is obtained by acting with the operator $\mathbb{L}(2,3,4,5)$ :

$$
\mathcal{N}_{6}([[[2,3], 4], 5], v):={ }^{2}=\mathbb{L}(2,3,4,5) \circ \mathcal{N}_{6}(2345, v) \text {. }
$$

The BCJ numerator thus obtained does not contain any free parameter and is unique:

$$
\begin{aligned}
& \mathcal{N}_{6}([[[2,3], 4], 5], v) \\
& =8 m \mathbb{L}(2,3,4,5) \circ\left[\frac{v \cdot F_{2} \cdot F_{3} \cdot V_{3} \cdot F_{4} \cdot F_{5} \cdot v}{2 v \cdot p_{3} v \cdot p_{45}}+\frac{v \cdot F_{2} \cdot F_{3} \cdot V_{3} \cdot F_{4} \cdot V_{4} \cdot F_{5} \cdot v}{2 v \cdot p_{3} v \cdot p_{5} v \cdot p_{45}}+\frac{v \cdot F_{4} \cdot F_{5} \cdot V_{3} \cdot F_{2} \cdot V_{4} \cdot F_{3} \cdot v}{2 v \cdot p_{3} v \cdot p_{4} v \cdot p_{345}}\right. \\
& \left.-\frac{v \cdot F_{3} \cdot F_{5} \cdot V_{3} \cdot F_{2} \cdot V_{3} \cdot F_{4} \cdot v}{2 v \cdot p_{3} v \cdot p_{4} v \cdot p_{345}}-\frac{v \cdot F_{3} \cdot F_{4} \cdot V_{3} \cdot F_{2} \cdot V_{3} \cdot F_{5} \cdot v}{2 v \cdot p_{3} v \cdot p_{5} v \cdot p_{345}}-\frac{v \cdot p_{45} v \cdot F_{3} \cdot F_{4} \cdot V_{3} \cdot F_{2} \cdot V_{4} \cdot F_{5} \cdot v}{2 v \cdot p_{3} v \cdot p_{4} v \cdot p_{5} v \cdot p_{345}}\right] .
\end{aligned}
$$

The colour-ordered amplitude in the novel colour-kinematics duality form is then

$$
\begin{aligned}
A_{6}^{\mathrm{YM}-\mathrm{M}}(123456)= & \frac{\mathcal{N}_{6}([[[2,3], 4], 5], v)}{s_{2345} s_{234} s_{23}}+\frac{\mathcal{N}_{6}([2,[3,[4,5]]], v)}{s_{2345} s_{345} s_{45}}+\frac{\mathcal{N}_{6}([[2,3],[4,5]], v)}{s_{2345} s_{23} s_{45}} \\
& +\frac{\mathcal{N}_{6}([[2,[3,4]], 5], v)}{s_{2345} s_{234} s_{34}}+\frac{\mathcal{N}_{6}([2,[[3,4], 5]], v)}{s_{2345} s_{34} s_{345}}
\end{aligned}
$$

The gravity amplitude is constructed similarly from the double copy:

$$
\begin{aligned}
A_{6}^{\mathrm{GR}-\mathrm{M}}(123456)= & \frac{\left[\mathcal{N}_{6}([[2,3],[4,5]], v)\right]^{2}}{s_{23} s_{45} s_{2345}}+\frac{\left[\mathcal{N}_{6}([2,[3,[4,5]]], v)\right]^{2}}{s_{345} s_{45} s_{2345}}+\frac{\left[\mathcal{N}_{6}([[2,4],[3,5]], v)\right]^{2}}{s_{24} s_{35} s_{2345}} \\
& +\frac{\left[\mathcal{N}_{6}([2,[4,[3,5]]], v)\right]^{2}}{s_{345} s_{35} s_{2345}}+\frac{\left[\mathcal{N}_{6}([[2,5],[3,4]], v)\right]^{2}}{s_{25} s_{34} s_{2345}}+\frac{\left[\mathcal{N}_{6}([2,[5,[3,4]]], v)\right]^{2}}{s_{345} s_{34} s_{2345}} \\
& +\frac{\left[\mathcal{N}_{6}([3,[2,[4,5]]], v)\right]^{2}}{s_{245} s_{45} s_{2345}}+\frac{\left[\mathcal{N}_{6}([3,[4,[2,5]]], v)\right]^{2}}{s_{245} s_{25} s_{2345}}+\frac{\left[\mathcal{N}_{6}([3,[5,[2,4]]], v)\right]^{2}}{s_{24} s_{245} s_{2345}} \\
& +\frac{\left[\mathcal{N}_{6}([4,[2,[3,5]]], v)\right]^{2}}{s_{235} s_{35} s_{2345}}+\frac{\left[\mathcal{N}_{6}([4,[3,[2,5]]], v)\right]^{2}}{s_{235} s_{25} s_{2345}}+\frac{\left[\mathcal{N}_{6}([4,[5,[2,3]]], v)\right]^{2}}{s_{23} s_{235} s_{2345}} \\
& +\frac{\left[\mathcal{N}_{6}([5,[2,[3,4]]], v)\right]^{2}}{s_{234} s_{34} s_{2345}}+\frac{\left[\mathcal{N}_{6}([5,[3,[2,4]]], v)\right]^{2}}{s_{24} s_{234} s_{2345}}+\frac{\left[\mathcal{N}_{6}([5,[4,[2,3]]], v)\right]^{2}}{s_{23} s_{234} s_{2345}} \\
= & \frac{\left[\mathcal{N}_{6}([[2,3],[4,5]], v)\right]^{2}}{8 s_{23} s_{45} s_{2345}}+\frac{\left[\mathcal{N}_{6}([2,[3,[4,5]]], v)\right]^{2}}{2 s_{345} s_{45} s_{2345}}+\text { permutations of } 2,3,4,5 .
\end{aligned}
$$

Note that gauge invariance, crossing symmetry and locality for the massless particles in the BCJ numerator (5.24) are manifest.

\section{Local BCJ numerators for pure Yang-Mills theory}

The numerators we have constructed in the HEFT are closely related to the local BCJ numerators for pure Yang-Mills theory by the following equation

$$
N_{n-1}^{\mathrm{YM}}(234 \cdots(n-1) 1)=\left.\mathcal{N}_{n}([\cdots[[2,3], 4], \cdots, n-1], v)\right|_{v \rightarrow \varepsilon_{1}, p_{234 \cdots n-1}^{2}=0} .
$$


We have checked this relation at five and six points, and we will now illustrate it in detail in the five-point case.

At five points, the main observation is to impose the on-shell condition for the leg with momentum $p_{234}$. Doing so, we get

$$
N_{4}^{\mathrm{YM}}(2341)=\left.\mathcal{N}_{5}([[2,3], 4], v)\right|_{m v \rightarrow \varepsilon_{1}, p_{234}^{2}=0} .
$$

We expand $\mathcal{N}_{5}([[2,3], 4], v)$ in $(5.13)$ and get

$$
\begin{aligned}
N_{4}^{\mathrm{YM}}(2341)= & 4\left(\varepsilon_{1} \cdot \varepsilon_{4} \varepsilon_{2} \cdot p_{3} \varepsilon_{3} \cdot p_{4}-\varepsilon_{1} \cdot \varepsilon_{4} \varepsilon_{2} \cdot p_{4} \varepsilon_{3} \cdot p_{2}+\varepsilon_{2} \cdot \varepsilon_{4} \varepsilon_{1} \cdot p_{4} \varepsilon_{3} \cdot p_{2}+\varepsilon_{1} \cdot \varepsilon_{2} \varepsilon_{4} \cdot p_{23} \varepsilon_{3} \cdot p_{2}\right. \\
& -\varepsilon_{3} \cdot \varepsilon_{4} \varepsilon_{1} \cdot p_{4} \varepsilon_{2} \cdot p_{3}-\varepsilon_{1} \cdot \varepsilon_{3} \varepsilon_{2} \cdot p_{3} \varepsilon_{4} \cdot p_{23}+\varepsilon_{2} \cdot \varepsilon_{3} \varepsilon_{1} \cdot p_{3} \varepsilon_{4} \cdot p_{23}+\varepsilon_{2} \cdot \varepsilon_{3} \varepsilon_{1} \cdot p_{4} \varepsilon_{4} \cdot p_{3} \\
& \left.+p_{2} \cdot p_{34} \varepsilon_{1} \cdot \varepsilon_{4} \varepsilon_{2} \cdot \varepsilon_{3}-\frac{1}{4} s_{23} \varepsilon_{1} \cdot \varepsilon_{2} \varepsilon_{3} \cdot \varepsilon_{4}+\frac{1}{2} s_{23} \mathcal{I}(3,4)\right)
\end{aligned}
$$

where

$$
\begin{aligned}
\mathcal{I}(3,4)= & -\frac{1}{2} \frac{\varepsilon_{1} \cdot \varepsilon_{2} \varepsilon_{3} \cdot \varepsilon_{4}}{\varepsilon_{1} \cdot p_{34}}\left(\varepsilon_{1} \cdot p_{4}-\varepsilon_{1} \cdot p_{3}\right)+\frac{\varepsilon_{1} \cdot \varepsilon_{2} \varepsilon_{1} \cdot \varepsilon_{3} \varepsilon_{1} \cdot \varepsilon_{4}}{\varepsilon_{1} \cdot p_{34}}\left(\frac{s_{24}}{\varepsilon_{1} \cdot p_{3}}-\frac{s_{23}}{\varepsilon_{1} \cdot p_{4}}\right) \\
& +\varepsilon_{1} \cdot \varepsilon_{3} \varepsilon_{1} \cdot \varepsilon_{4}\left(\frac{\varepsilon_{2} \cdot p_{4}}{\varepsilon_{1} \cdot p_{3}}-\frac{\varepsilon_{2} \cdot p_{3}}{\varepsilon_{1} \cdot p_{4}}\right)+\frac{\varepsilon_{1} \cdot \varepsilon_{2}}{\varepsilon_{1} \cdot p_{34}}\left(\varepsilon_{1} \cdot \varepsilon_{4} \varepsilon_{3} \cdot p_{4}-\varepsilon_{1} \cdot \varepsilon_{3} \varepsilon_{4} \cdot p_{3}\right) \\
& +\frac{\varepsilon_{1} \cdot \varepsilon_{4} \varepsilon_{2} \cdot \varepsilon_{3} \varepsilon_{1} \cdot p_{3}}{\varepsilon_{1} \cdot p_{4}}+\frac{\varepsilon_{1} \cdot \varepsilon_{2} \varepsilon_{1} \cdot \varepsilon_{4} \varepsilon_{3} \cdot p_{2}}{\varepsilon_{1} \cdot p_{4}}-\frac{\varepsilon_{1} \cdot \varepsilon_{3} \varepsilon_{2} \cdot \varepsilon_{4} \varepsilon_{1} \cdot p_{4}}{\varepsilon_{1} \cdot p_{3}}-\frac{\varepsilon_{1} \cdot \varepsilon_{2} \varepsilon_{1} \cdot \varepsilon_{3} \varepsilon_{4} \cdot p_{2}}{\varepsilon_{1} \cdot p_{3}} .
\end{aligned}
$$

As $\frac{1}{2} \mathbb{L}(3,4) \circ \mathcal{I}(3,4)=\mathcal{I}(3,4)$, then $s_{23} \mathcal{I}(3,4)$ is a pure gauge term ${ }^{7}$ which does not contribute to the Yang-Mills amplitude. By removing pure gauge terms we arrive at an expression which is manifestly local,

$$
\begin{aligned}
N_{4}^{\mathrm{YM}}(2341)= & 4\left(\varepsilon_{1} \cdot \varepsilon_{4} \varepsilon_{2} \cdot p_{3} \varepsilon_{3} \cdot p_{4}-\varepsilon_{1} \cdot \varepsilon_{4} \varepsilon_{2} \cdot p_{4} \varepsilon_{3} \cdot p_{2}+\varepsilon_{2} \cdot \varepsilon_{4} \varepsilon_{1} \cdot p_{4} \varepsilon_{3} \cdot p_{2}+\varepsilon_{1} \cdot \varepsilon_{2} \varepsilon_{4} \cdot p_{23} \varepsilon_{3} \cdot p_{2}\right. \\
& -\varepsilon_{3} \cdot \varepsilon_{4} \varepsilon_{1} \cdot p_{4} \varepsilon_{2} \cdot p_{3}-\varepsilon_{1} \cdot \varepsilon_{3} \varepsilon_{2} \cdot p_{3} \varepsilon_{4} \cdot p_{23}+\varepsilon_{2} \cdot \varepsilon_{3} \varepsilon_{1} \cdot p_{3} \varepsilon_{4} \cdot p_{23}+\varepsilon_{2} \cdot \varepsilon_{3} \varepsilon_{1} \cdot p_{4} \varepsilon_{4} \cdot p_{3} \\
& \left.+p_{2} \cdot p_{34} \varepsilon_{1} \cdot \varepsilon_{4} \varepsilon_{2} \cdot \varepsilon_{3}-\frac{1}{4} s_{23} \varepsilon_{1} \cdot \varepsilon_{2} \varepsilon_{3} \cdot \varepsilon_{4}\right) .
\end{aligned}
$$

Finally, we have checked that the numerator (6.5) generates the correct amplitude. This numerator by itself is no longer gauge invariant.

We have also confirmed that the six-point numerator in HEFT is directly related to the five-point BCJ numerator in pure Yang-Mills theory,

$$
N_{5}^{\mathrm{YM}}(23451)=\left.\mathcal{N}_{6}([[[2,3], 4], 5], v)\right|_{v \rightarrow \varepsilon_{1}, p_{2345}^{2}=0} \cdot
$$

This result is in agreement with [62].

\footnotetext{
${ }^{7}$ We comment that pure gauge terms are classified by the $\mathbb{L}$-invariants $\mathcal{I}$ as discussed in [63].
} 


\section{Conclusions}

We conclude by summarising a number of interesting properties of the new BCJ numerators we have constructed in the HEFT:

1. Gauge invariance. We have expressed all the BCJ numerators $\mathcal{N}_{n}(\Gamma, v)$ in terms of field strengths, hence they are gauge invariant even if the on-shell and transversality conditions $p_{i} \cdot \varepsilon_{i}=0$ are not imposed.

2. Locality with respect to the massless particles. Our new BCJ numerators do not contain spurious poles. This is particularly convenient when constructing loop integrands via generalised unitarity, as we will demonstrate in several examples at loop level in [81].

3. Crossing symmetry and Jacobi relation are manifest. As all the BCJ numerators are generated from the pre-numerator, the crossing symmetry with respect to all the massless particles is manifest. Moreover, based on the assumption of associativity of the fusion product, the Jacobi relations are automatically satisfied by the BCJ numerators.

4. Numerators for pure Yang-Mills theory. Our BCJ numerators are directly related to local BCJ numerators for pure Yang-Mills theory.

Note that usually BCJ numerators are manifestly local but not gauge invariant, while using KLT relations one arrives at expressions which are gauge invariant but non-local; here we have both locality and gauge invariance. We have checked the above properties up to six particles, and we conjecture them to be valid for arbitrary number of particles.

There are a few directions for future work. First, it would be desirable to find a proof of the gauge invariance of the BCJ numerators for any multiplicity. Is this a property of the HEFT or only of its leading term considered in this paper? A pressing question is to find a closed form of the pre-numerator, which would require a full understanding of the fusion rule for any number of particles. Finally, it would also be important to understand the subleading terms in the inverse mass expansion as well as higher-spin effects, to ascertain how much of the structures we have uncovered survive the expansion.

\section{Acknowledgments}

We would like to thank Henrik Johansson, Rodolfo Russo, Fei Teng and Tianheng Wang for interesting discussions. This work was supported by the Science and Technology Facilities Council (STFC) Consolidated Grants ST/P000754/1 "String theory, gauge theory 8 duality" and ST/T000686/1 "Amplitudes, strings \& duality" and by the European Union's Horizon 2020 research and innovation programme under the Marie Skłodowska-Curie grant agreement No. 764850 "SAGEX". CW is supported by a Royal Society University Research Fellowship No. UF160350. 
Open Access. This article is distributed under the terms of the Creative Commons Attribution License (CC-BY 4.0), which permits any use, distribution and reproduction in any medium, provided the original author(s) and source are credited.

\section{References}

[1] H. Georgi, An Effective Field Theory for Heavy Quarks at Low-energies, Phys. Lett. B 240 (1990) 447 [INSPIRE].

[2] M.E. Luke and A.V. Manohar, Reparametrization invariance constraints on heavy particle effective field theories, Phys. Lett. B 286 (1992) 348 [hep-ph/9205228] [INSPIRE].

[3] M. Neubert, Heavy quark symmetry, Phys. Rept. 245 (1994) 259 [hep-ph/9306320] [INSPIRE].

[4] A.V. Manohar and M.B. Wise, Heavy quark physics, Camb. Monogr. Part. Phys. Nucl. Phys. Cosmol. 10 (2000) 1 [INSPIRE].

[5] D.A. Kosower, B. Maybee and D. O'Connell, Amplitudes, Observables, and Classical Scattering, JHEP 02 (2019) 137 [arXiv:1811.10950] [INSPIRE].

[6] P.H. Damgaard, K. Haddad and A. Helset, Heavy Black Hole Effective Theory, JHEP 11 (2019) 070 [arXiv: 1908.10308] [inSPIRE].

[7] D. Neill and I.Z. Rothstein, Classical Space-Times from the S Matrix, Nucl. Phys. B $\mathbf{8 7 7}$ (2013) 177 [arXiv: 1304.7263] [INSPIRE].

[8] N.E.J. Bjerrum-Bohr, J.F. Donoghue and P. Vanhove, On-shell Techniques and Universal Results in Quantum Gravity, JHEP 02 (2014) 111 [arXiv:1309.0804] [INSPIRE].

[9] N.E.J. Bjerrum-Bohr, J.F. Donoghue, B.R. Holstein, L. Planté and P. Vanhove, Bending of Light in Quantum Gravity, Phys. Rev. Lett. 114 (2015) 061301 [arXiv:1410.7590] [INSPIRE].

[10] N.E.J. Bjerrum-Bohr, J.F. Donoghue, B.R. Holstein, L. Plante and P. Vanhove, Light-like Scattering in Quantum Gravity, JHEP 11 (2016) 117 [arXiv:1609.07477] [INSPIRE].

[11] F. Cachazo and A. Guevara, Leading Singularities and Classical Gravitational Scattering, JHEP 02 (2020) 181 [arXiv:1705.10262] [INSPIRE].

[12] A. Guevara, Holomorphic Classical Limit for Spin Effects in Gravitational and Electromagnetic Scattering, JHEP 04 (2019) 033 [arXiv: 1706.02314] [INSPIRE].

[13] H.-H. Chi, Graviton Bending in Quantum Gravity from One-Loop Amplitudes, Phys. Rev. D 99 (2019) 126008 [arXiv: 1903.07944] [INSPIRE].

[14] A. Brandhuber and G. Travaglini, On higher-derivative effects on the gravitational potential and particle bending, JHEP 01 (2020) 010 [arXiv: 1905.05657] [INSPIRE].

[15] W.T. Emond and N. Moynihan, Scattering Amplitudes, Black Holes and Leading Singularities in Cubic Theories of Gravity, JHEP 12 (2019) 019 [arXiv: 1905. 08213] [INSPIRE].

[16] M. Accettulli Huber, A. Brandhuber, S. De Angelis and G. Travaglini, Eikonal phase matrix, deflection angle and time delay in effective field theories of gravity, Phys. Rev. D 102 (2020) 046014 [arXiv:2006.02375] [INSPIRE].

[17] Z. Bern, C. Cheung, R. Roiban, C.-H. Shen, M.P. Solon and M. Zeng, Scattering Amplitudes and the Conservative Hamiltonian for Binary Systems at Third Post-Minkowskian Order, Phys. Rev. Lett. 122 (2019) 201603 [arXiv:1901.04424] [INSPIRE]. 
[18] Z. Bern, C. Cheung, R. Roiban, C.-H. Shen, M.P. Solon and M. Zeng, Black Hole Binary Dynamics from the Double Copy and Effective Theory, JHEP 10 (2019) 206 [arXiv: 1908.01493] [INSPIRE].

[19] C. Cheung and M.P. Solon, Classical gravitational scattering at $\mathcal{O}\left(G^{3}\right)$ from Feynman diagrams, JHEP 06 (2020) 144 [arXiv:2003.08351] [INSPIRE].

[20] Z. Bern et al., Scattering Amplitudes and Conservative Binary Dynamics at $\mathcal{O}\left(G^{4}\right)$, Phys. Rev. Lett. 126 (2021) 171601 [arXiv:2101.07254] [INSPIRE].

[21] Z. Bern, J.J.M. Carrasco and H. Johansson, New Relations for Gauge-Theory Amplitudes, Phys. Rev. D 78 (2008) 085011 [arXiv:0805.3993] [INSPIRE].

[22] Z. Bern, J.J.M. Carrasco and H. Johansson, Perturbative Quantum Gravity as a Double Copy of Gauge Theory, Phys. Rev. Lett. 105 (2010) 061602 [arXiv:1004.0476] [InSPIRE].

[23] Z. Bern, J.J. Carrasco, M. Chiodaroli, H. Johansson and R. Roiban, The Duality Between Color and Kinematics and its Applications, arXiv:1909.01358 [INSPIRE].

[24] T. Bargheer, S. He and T. McLoughlin, New Relations for Three-Dimensional Supersymmetric Scattering Amplitudes, Phys. Rev. Lett. 108 (2012) 231601 [arXiv: 1203.0562] [INSPIRE].

[25] J. Broedel and L.J. Dixon, Color-kinematics duality and double-copy construction for amplitudes from higher-dimension operators, JHEP 10 (2012) 091 [arXiv:1208.0876] [INSPIRE].

[26] M. Chiodaroli, Q. Jin and R. Roiban, Color/kinematics duality for general abelian orbifolds of $N=4$ super Yang-Mills theory, JHEP 01 (2014) 152 [arXiv:1311.3600] [INSPIRE].

[27] H. Johansson and A. Ochirov, Pure Gravities via Color-Kinematics Duality for Fundamental Matter, JHEP 11 (2015) 046 [arXiv: 1407.4772] [INSPIRE].

[28] M. Chiodaroli, M. Günaydin, H. Johansson and R. Roiban, Scattering amplitudes in $\mathcal{N}=2$ Maxwell-Einstein and Yang-Mills/Einstein supergravity, JHEP 01 (2015) 081 [arXiv: 1408.0764] [INSPIRE].

[29] M. Chiodaroli, M. Günaydin, H. Johansson and R. Roiban, Spontaneously Broken Yang-Mills-Einstein Supergravities as Double Copies, JHEP 06 (2017) 064 [arXiv: 1511.01740] [INSPIRE].

[30] H. Johansson and J. Nohle, Conformal Gravity from Gauge Theory, arXiv:1707.02965 [INSPIRE].

[31] M. Chiodaroli, M. Günaydin, H. Johansson and R. Roiban, Non-Abelian gauged supergravities as double copies, JHEP 06 (2019) 099 [arXiv: 1812.10434] [INSPIRE].

[32] G. Chen and Y.-J. Du, Amplitude Relations in Non-linear Sigma Model, JHEP 01 (2014) 061 [arXiv:1311.1133] [INSPIRE].

[33] J.J.M. Carrasco, C.R. Mafra and O. Schlotterer, Abelian Z-theory: NLSM amplitudes and $\alpha$ '-corrections from the open string, JHEP 06 (2017) 093 [arXiv:1608.02569] [INSPIRE].

[34] C.R. Mafra and O. Schlotterer, Non-abelian Z-theory: Berends-Giele recursion for the $\alpha^{\prime}$-expansion of disk integrals, JHEP 01 (2017) 031 [arXiv: 1609.07078] [INSPIRE].

[35] J.J.M. Carrasco, C.R. Mafra and O. Schlotterer, Semi-abelian Z-theory: NLSM+ $\phi^{3}$ from the open string, JHEP 08 (2017) 135 [arXiv:1612.06446] [INSPIRE]. 
[36] C. Cheung and C.-H. Shen, Symmetry for Flavor-Kinematics Duality from an Action, Phys. Rev. Lett. 118 (2017) 121601 [arXiv: 1612.00868] [INSPIRE].

[37] C. Cheung, C.-H. Shen and C. Wen, Unifying Relations for Scattering Amplitudes, JHEP 02 (2018) 095 [arXiv: 1705.03025] [INSPIRE].

[38] C. Cheung, G.N. Remmen, C.-H. Shen and C. Wen, Pions as Gluons in Higher Dimensions, JHEP 04 (2018) 129 [arXiv:1709.04932] [InSPIRE].

[39] H. Kawai, D.C. Lewellen and S.H.H. Tye, A Relation Between Tree Amplitudes of Closed and Open Strings, Nucl. Phys. B 269 (1986) 1 [INSPIRE].

[40] N.E.J. Bjerrum-Bohr, P.H. Damgaard, T. Sondergaard and P. Vanhove, The Momentum Kernel of Gauge and Gravity Theories, JHEP 01 (2011) 001 [arXiv:1010.3933] [InSPIRE].

[41] C.R. Mafra, O. Schlotterer and S. Stieberger, Explicit BCJ Numerators from Pure Spinors, JHEP 07 (2011) 092 [arXiv: 1104.5224] [INSPIRE].

[42] C.-H. Fu, Y.-J. Du and B. Feng, An algebraic approach to BCJ numerators, JHEP 03 (2013) 050 [arXiv: 1212.6168] [INSPIRE].

[43] C.R. Mafra and O. Schlotterer, Berends-Giele recursions and the BCJ duality in superspace and components, JHEP 03 (2016) 097 [arXiv:1510.08846] [INSPIRE].

[44] N.E.J. Bjerrum-Bohr, J.L. Bourjaily, P.H. Damgaard and B. Feng, Manifesting Color-Kinematics Duality in the Scattering Equation Formalism, JHEP 09 (2016) 094 [arXiv: 1608.00006] [INSPIRE].

[45] Y.-J. Du and F. Teng, BCJ numerators from reduced Pfaffian, JHEP 04 (2017) 033 [arXiv: 1703.05717] [INSPIRE].

[46] G. Chen and T. Wang, BCJ Numerators from Differential Operator of Multidimensional Residue, Eur. Phys. J. C 80 (2020) 37 [arXiv:1709. 08503] [InSPIRE].

[47] C.-H. Fu, P. Vanhove and Y. Wang, A Vertex Operator Algebra Construction of the Colour-Kinematics Dual numerator, JHEP 09 (2018) 141 [arXiv: 1806.09584] [INSPIRE].

[48] E. Bridges and C.R. Mafra, Algorithmic construction of SYM multiparticle superfields in the BCJ gauge, JHEP 10 (2019) 022 [arXiv: 1906.12252] [INSPIRE].

[49] Z. Bern, J.J.M. Carrasco, L.J. Dixon, H. Johansson and R. Roiban, Simplifying Multiloop Integrands and Ultraviolet Divergences of Gauge Theory and Gravity Amplitudes, Phys. Rev. D 85 (2012) 105014 [arXiv:1201.5366] [INSPIRE].

[50] N.E.J. Bjerrum-Bohr, T. Dennen, R. Monteiro and D. O'Connell, Integrand Oxidation and One-Loop Colour-Dual Numerators in N=4 Gauge Theory, JHEP 07 (2013) 092 [arXiv: 1303.2913] [INSPIRE].

[51] Z. Bern, S. Davies, T. Dennen, Y.-t. Huang and J. Nohle, Color-Kinematics Duality for Pure Yang-Mills and Gravity at One and Two Loops, Phys. Rev. D 92 (2015) 045041 [arXiv:1303.6605] [INSPIRE].

[52] J. Nohle, Color-Kinematics Duality in One-Loop Four-Gluon Amplitudes with Matter, Phys. Rev. D 90 (2014) 025020 [arXiv: 1309.7416] [INSPIRE].

[53] G. Mogull and D. O'Connell, Overcoming Obstacles to Colour-Kinematics Duality at Two Loops, JHEP 12 (2015) 135 [arXiv:1511.06652] [INSPIRE].

[54] S. He, O. Schlotterer and Y. Zhang, New BCJ representations for one-loop amplitudes in gauge theories and gravity, Nucl. Phys. B 930 (2018) 328 [arXiv:1706.00640] [InSPIRE]. 
[55] H. Johansson, G. Kälin and G. Mogull, Two-loop supersymmetric QCD and half-maximal supergravity amplitudes, JHEP 09 (2017) 019 [arXiv:1706.09381] [INSPIRE].

[56] G. Kälin, G. Mogull and A. Ochirov, Two-loop $\mathcal{N}=2 S Q C D$ amplitudes with external matter from iterated cuts, JHEP 07 (2019) 120 [arXiv: 1811.09604] [INSPIRE].

[57] R.H. Boels, B.A. Kniehl, O.V. Tarasov and G. Yang, Color-kinematic Duality for Form Factors, JHEP 02 (2013) 063 [arXiv:1211.7028] [INSPIRE].

[58] G. Yang, Color-kinematics duality and Sudakov form factor at five loops for $N=4$ supersymmetric Yang-Mills theory, Phys. Rev. Lett. 117 (2016) 271602 [arXiv:1610.02394] [INSPIRE].

[59] R.H. Boels, T. Huber and G. Yang, The Sudakov form factor at four loops in maximal super Yang-Mills theory, JHEP 01 (2018) 153 [arXiv:1711.08449] [INSPIRE].

[60] R. Monteiro and D. O'Connell, The Kinematic Algebra From the Self-Dual Sector, JHEP 07 (2011) 007 [arXiv:1105.2565] [INSPIRE].

[61] N.E.J. Bjerrum-Bohr, P.H. Damgaard, R. Monteiro and D. O'Connell, Algebras for Amplitudes, JHEP 06 (2012) 061 [arXiv:1203.0944] [INSPIRE].

[62] G. Chen, H. Johansson, F. Teng and T. Wang, On the kinematic algebra for BCJ numerators beyond the MHV sector, JHEP 11 (2019) 055 [arXiv: 1906.10683] [INSPIRE].

[63] G. Chen, H. Johansson, F. Teng and T. Wang, Next-to-MHV Yang-Mills kinematic algebra, arXiv:2104.12726 [INSPIRE].

[64] C.-H. Fu and K. Krasnov, Colour-Kinematics duality and the Drinfeld double of the Lie algebra of diffeomorphisms, JHEP 01 (2017) 075 [arXiv: 1603.02033] [INSPIRE].

[65] M. Reiterer, A homotopy BV algebra for Yang-Mills and color-kinematics, arXiv:1912.03110 [INSPIRE].

[66] M. Tolotti and S. Weinzierl, Construction of an effective Yang-Mills Lagrangian with manifest BCJ duality, JHEP 07 (2013) 111 [arXiv:1306.2975] [INSPIRE].

[67] S. Mizera, Kinematic Jacobi Identity is a Residue Theorem: Geometry of Color-Kinematics Duality for Gauge and Gravity Amplitudes, Phys. Rev. Lett. 124 (2020) 141601 [arXiv: 1912.03397] [INSPIRE].

[68] L. Borsten, B. Jurčo, H. Kim, T. Macrelli, C. Sämann and M. Wolf, Becchi-Rouet-Stora-Tyutin-Lagrangian Double Copy of Yang-Mills Theory, Phys. Rev. Lett. 126 (2021) 191601 [arXiv:2007.13803] [INSPIRE].

[69] L. Borsten and S. Nagy, The pure BRST Einstein-Hilbert Lagrangian from the double-copy to cubic order, JHEP 07 (2020) 093 [arXiv: 2004.14945] [INSPIRE].

[70] L. Borsten, H. Kim, B. Jurčo, T. Macrelli, C. Sämann and M. Wolf, Double Copy from Homotopy Algebras, arXiv:2102.11390 [INSPIRE].

[71] P. Ferrero and D. Francia, On the Lagrangian formulation of the double copy to cubic order, JHEP 02 (2021) 213 [arXiv:2012.00713] [INSPIRE].

[72] H. Johansson and A. Ochirov, Color-Kinematics Duality for QCD Amplitudes, JHEP 01 (2016) 170 [arXiv: 1507.00332] [InSPIRE].

[73] L. de la Cruz, A. Kniss and S. Weinzierl, Proof of the fundamental BCJ relations for QCD amplitudes, JHEP 09 (2015) 197 [arXiv:1508.01432] [INSPIRE]. 
[74] A. Luna, I. Nicholson, D. O'Connell and C.D. White, Inelastic Black Hole Scattering from Charged Scalar Amplitudes, JHEP 03 (2018) 044 [arXiv:1711.03901] [INSPIRE].

[75] R.W. Brown and S.G. Naculich, KLT-type relations for QCD and bicolor amplitudes from color-factor symmetry, JHEP 03 (2018) 057 [arXiv: 1802.01620] [INSPIRE].

[76] J. Plefka and W. Wormsbecher, New relations for graviton-matter amplitudes, Phys. Rev. D 98 (2018) 026011 [arXiv: 1804.09651] [INSPIRE].

[77] H. Johansson and A. Ochirov, Double copy for massive quantum particles with spin, JHEP 09 (2019) 040 [arXiv: 1906.12292] [INSPIRE].

[78] Y.F. Bautista and A. Guevara, On the Double Copy for Spinning Matter, arXiv:1908.11349 [INSPIRE].

[79] J. Plefka, C. Shi and T. Wang, Double copy of massive scalar QCD, Phys. Rev. D 101 (2020) 066004 [arXiv:1911.06785] [INSPIRE].

[80] K. Haddad and A. Helset, The double copy for heavy particles, Phys. Rev. Lett. 125 (2020) 181603 [arXiv: 2005.13897] [INSPIRE].

[81] A. Brandhuber, G. Chen, G. Travaglini and C. Wen, Classical gravitational scattering from a gauge-invariant double copy, to appear.

[82] L. Heisenberg, A systematic approach to generalisations of General Relativity and their cosmological implications, Phys. Rept. 796 (2019) 1 [arXiv:1807.01725] [INSPIRE].

[83] O. Minazzoli, Conservation laws in theories with universal gravity/matter coupling, Phys. Rev. D 88 (2013) 027506 [arXiv:1307.1590] [INSPIRE].

[84] E.R. Huggins, Quantum mechanics of the interaction of gravity with electrons: theory of a spin-two field coupled to energy, Ph.D. Thesis, California Institute of Technology (1962).

[85] S. Sannan, Gravity as the Limit of the Type II Superstring Theory, Phys. Rev. D 34 (1986) 1749 [INSPIRE].

[86] M. Linckelmann, The block theory of finite group algebras, vol. 1, Cambridge University Press (2018) [DOI].

[87] T.W. Hungerford, Algebra, Graduate Texts in Mathematics, vol. 73, Springer-Verlag New York, 1st ed. (1980) [DOI].

[88] C. Reutenauer, Free Lie algebras, Clarendon Press (1993). 